Review Article

\title{
Osteoporosis in Chronic Obstructive Pulmonary Disease: More than just a Comorbidity
}

\author{
Mradul Kumar Daga', Naresh Kumar ${ }^{2}$, Raghu RV ${ }^{3}$, Govind Mawari ${ }^{4}$, Siddharth Chand $^{5}$,
} J Aarthi ${ }^{6}$, Manish Kumar Jha $^{7}$, Maryam Hussain $^{8}$

${ }^{1}$ Director Professor, ${ }^{3,5,6}$ Post Graduate, ${ }^{8}$ Department of Medicine, Maulana Azad Medical College, New Delhi, India. ${ }^{2}$ Professor of Medicine \& Head, Pulmonary Medicine, Maulana Azad Medical College, New Delhi, India.

${ }^{4}$ Scientist - C, Centre for Occupational and Environmental Health, Maulana Azad Medical College, New Delhi, India. ${ }^{7}$ Research Associate, Centre for Occupational and Environmental Health, Maulana Azad Medical College, New Delhi, India. DOI: https://doi.org/10.24321/2349.7181.202011

I $\begin{array}{llllll}\mathbf{N} & \mathbf{F} & \mathbf{O}\end{array}$

Corresponding Author:

Mradul Kumar Daga, Department of Medicine, Maulana Azad Medical College, New Delhi, India. E-mail Id:

drmraduldaga@gmail.com

Orcid Id:

https://orcid.org/0000-0001-7774-7602

How to cite this article:

Daga MK, Kumar N, Raghu RV, Mawari G, Chand $\mathrm{S}$, Aarthi J. Osteoporosis in Chronic Obstructive Pulmonary Disease: More than just a Comorbidity. J Adv Res Med 2020; 7(3): 7-12.

Date of Submission: 2020-11-04

Date of Acceptance: 2020-12-20
$\begin{array}{llllllll}\mathbf{A} & \mathbf{B} & \mathbf{S} & \mathbf{T} & \mathbf{R} & \mathbf{A} & \mathbf{C} & \mathbf{T}\end{array}$

Chronic Obstructive Pulmonary Disease (COPD) is one of the leading causes of mortality and loss of Disability-Adjusted Life-Years (DALYs) worldwide. It often is accompanied by the presence of various systemic comorbidities including osteoporosis which may have an impact on the course of the disease. Osteopenia and osteoporosis are the consequences of loss of Bone Mineral Density (BMD) and have been widely known major comorbidities in COPD patients.

Female sex, age, and smoking are common pathogenic factors for both COPD and osteoporosis, other factors such as reduced daily physical activity, malnutrition, low body mass index, hypogonadism, vitamin D deficiency, chronic renal insufficiency, chronic hypoxemia, and drugs like corticosteroids, have been invoked to explain such a frequent association between them. Osteoporosis in COPD is however often undertreated. It has been shown in recent studies that both decreased Bone Mineral Density (BMD) and impaired bone quality contribute to bone fragility, causing fractures in COPD patients. Pulmonary function and activities of the daily life of COPD patients may be further deteriorated by osteoporosis-associated fractures. Calcium and vitamin $\mathrm{D}$, hormone replacement when indicated, calcitonin, and bisphosphonate administration are few effective strategies to tackle bone loss and osteoporosis. Awareness about this high prevalence of osteoporosis in COPD patients is critically important and physicians should look for such fracture risks. Routine screening and early diagnosis of osteoporosis will enable physicians to provide the appropriate treatment to prevent fracture, which leads to improved quality of life as well as better long-term prognosis.

Keywords: COPD, Osteoporosis, Bone Mineral Density (BMD), Vertebral Fracture, DEXA, FRAX 


\section{Introduction}

Chronic Obstructive Pulmonary Disease (COPD) is often described as a multisystem disease, characterized by pulmonary and extrapulmonary consequences. ${ }^{1-3}$ Systemic inflammation, inactivity, deconditioning and continued smoking are few suggested mechanisms for the development of extrapulmonary consequences or comorbidities in COPD. ${ }^{4}$ Cigarette smoking, by far the main causative factor in chronic obstructive pulmonary disease, contributes to the increased risk of respiratory and many other nonrespiratory conditions often associated with aging.

Osteoporosis is one of the major systemic comorbidities associated with COPD. Osteoporosis is characterized by reduced skeletal resistance secondary to the deterioration of the microarchitecture of bone tissue, leading to reduced bone mass and lower mineral content. The consequences are increased fracture risk and bone fragility. Osteoporosis is defined as bone mineral density $\geq 2.5 \mathrm{SD}$ below the mean for young adults (T-score $\mathrm{f}-2.5$ ), as measured by bone densitometry. The spine, hip, and wrist are the preferred site for osteoporosis-related fracture. Osteopenia is the term to a "preclinical" stage of osteoporosis and is defined as a T-score between -2.5 and -1 . Osteoporosis is the expected comorbidity in COPD as both are disorders of the second half of life. The pre-existing physical inactivity and risk of vertebral fractures in patients of COPD are exacerbated by osteoporosis. With increasing number of vertebral fractures, lung capacity will reduce progressively. The vertebral fractures can also lead to kyphosis, which restricts respiratory movements and reduce lung function parameters.

\section{Epidemiology}

The prevalence of osteoporosis in COPD patients is greater than the general population. In a systematic review conducted by GRAAT-VERBBOM et al. they found that prevalence of osteoporosis and osteopenia varied from 9-69\% and $27-67 \%$, respectively based on the World Health Organization (WHO) definition and based on bone densitometry. ${ }^{5}$ The patients of COPD with osteoporosis are having significantly lower FEV and $\mathrm{BMI}$ values than the patients without osteoporosis. ${ }^{6}$

In a study conducted by SCANLON et al. a significant reduction in bone density over 3 years was seen in the triamcinolone treated group $(-1.66 \pm 4.4)$, compared to the placebo group $(0.1 \pm 4.04) .^{7}$ However, in another study changes in bone density and incidence of bone fracture were small and similar in all of the groups. ${ }^{8}$

MINEO et al. evaluated the bone density in 40 patients before and after 1 year of lung volume reduction surgery for emphysema and they found improved bone density in over half of the patients after the surgery despite oral steroid therapy. ${ }^{9}$
Even the asymptomatic vertebral fractures in COPD patients are of great concern, as they accelerate their declining lung function. The prevalence of vertebral fractures in an ambulatory COPD population was $41 \%$ and correlated with COPD severity as shown by the Evaluation of Obstructive Lung Disease and Osteoporosis (EOLO) study. ${ }^{10}$

\section{Pathophysiology}

Pathophysiological evidence supports the epidemiological observations and sheds light on the potential relationship between the two conditions.

Bone remodeling is regulated by three effectors at the cellular level:

- The osteoblasts that form the bone matrix and which is then calcified

- The osteoclasts that reabsorb bone

- The osteocytes that coordinate bone remodeling

Besides, three regulatory pathways control the interaction between osteoblasts and osteoclasts. The first involve direct interaction between these two cells via the receptor activator of NF-kB ligand (RANKL) present at the surface of osteoblasts and its receptor RANK at the surface of proosteoclasts, enabling the latter to differentiate into activated osteoclasts. The second pathway involved osteoprotegerin, a protein secreted by stromal cells and osteoblasts. It blocks the interaction between RANK and RANKL, reducing bone resorption. Finally, the $\mathrm{Wnt} / \mathrm{b}$-catenin regulatory pathway downstream of osteoblast activators remains poorly understood.

COPD and osteoporosis have shared common risk factors like smoking and systemic inflammation which affect RANK/ RANKL binding at the cellular level. Wnt can also be targeted by Inflammatory cytokines. Pro-inflammatory cytokines, such as IL-1b, IL-6, and TNF- $a$ and may promote bone resorption. ${ }^{11}$

Vitamin D improves the expression of the osteoprotegerin/ RANKL complex and hence reducing osteoclast formation. Through its effect on RANK/RANKL, vitamin D deficiency affects osteoclast maturation and stimulates Parathormone secretion. According to JANSSENS et al. the prevalence of vitamin D deficiency increases with the COPD stage and its around $77 \%$ for a very severe stage. ${ }^{12}$

It is a well established fact that oral steroid therapy will have harmful effects on bone metabolism and recent studies have highlighted the underlying mechanism and impact of oral steroid therapy on both bone resorption and impaired bone formation. By increasing the expression of RANKL, decreasing osteoprotegerin, and suppression of osteoclast apoptosis oral steroid affects bone homeostasis.

The hypogonadism which is common in COPD also plays a role in the development of osteoporosis. ${ }^{13}$ As seen in post- 
menopausal females, a drop in estrogen level inhibits the action of the osteoprotegerin/RANKL complex.

The lower levels of physical activity commonly seen in COPD patients also aggravate osteoporosis. Malnutrition which is a common finding in COPD especially in the advanced stage also affects bone metabolism significantly.

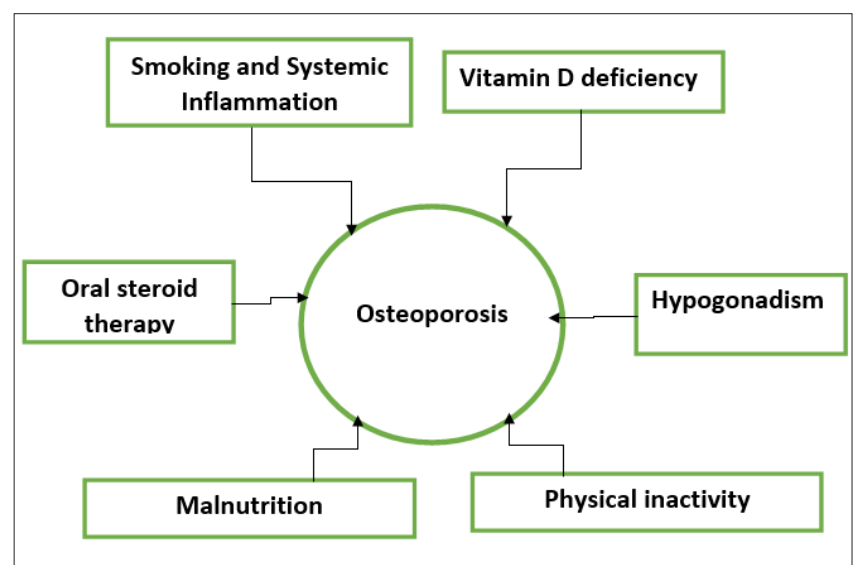

Figure 1.Pathophysiology of Osteoporosis in COPD Clinical Fractures in COPD

The low bone mass itself is asymptomatic and causes no morbidity. However, the consequences of osteoporosis, fractures, can cause significant debility. Thoracic vertebral fractures may further compromise lung function, and hip fractures decrease mobility and are associated with significant morbidity. ${ }^{14,15,16}$ Vertebral fractures are common in patients with COPD. In a study conducted in patients awaiting lung transplantation, the prevalence of vertebral fracture was as high as $29 \% .{ }^{17}$ Another study on COPD patients revealed the prevalence of vertebral fractures to be $49 \%$ in those who had never received any glucocorticoids to as high as $63 \%$ in those patients receiving systemic glucocorticoid therapy. ${ }^{18}$ Thoracic vertebral fractures were more common than lumbar fractures. Many cases of vertebral fractures may remain asymptomatic. However, both symptomatic and asymptomatic vertebral fractures can cause significant morbidity due to back pain and decreased functional performance. ${ }^{19,20}$ This pain can be distressing to the patient who is already struggling to breathe. Additionally, progressive kyphosis due to thoracic vertebral fractures may decrease lung volume, causing a restrictive ventilatory defect. ${ }^{14,21}$

The most serious consequence of osteoporosis is hip fracture. The risk for hip fracture varies inversely with Bone Mineral Density (BMD) and also increases with presence of one or more chronic conditions. ${ }^{22}$ Very limited data is available regarding the risk of hip fracture in COPD patients, however, the risk of hip fracture has been evaluated in oral corticosteroid users. The relative risk of hip fracture was found to be 1.61 ( $95 \% \mathrm{Cl}, 1.47$ to 1.76$)$ in a retrospective cohort study comparing all users of oral corticosteroids to subjects in a control group who had used topical steroids. ${ }^{23}$ Hip fractures have significant morbidity, often resulting in decreased mobility and independence for the patient. ${ }^{24}$ In severely dyspneic COPD patient, further loss of mobility after a hip fracture may lead to increased dependence on the caretaker.

\section{Assessment of Osteoporosis and Fracture Risk}

The fracture risk depends on bone strength, which is determined by BMD and bone quality. ${ }^{25}$ However, due to the absence of clinical tools for precise evaluation of bone quality, a diagnosis of osteoporosis has been reliant on BMD measurement. Several non-invasive techniques are available for estimating skeletal mass or BMD. They include Dual-Energy $x$-ray Absorptiometry (DEXA), Single energy $x$-ray absorptiometry (SXA), quantitative $C T$, and Ultrasound (US). DEXA is a highly accurate $x$-ray technique that has become the standard for measuring bone density. In a case-control study conducted by MK daga et al, in north India DEXA scan was used to find out the prevalence of osteoporosis and its severity in COPD patients and they found that the incidence of osteoporosis was significantly higher $(60 \%)$ in COPD patients as compared to healthy adults $(20 \%)$ with a p-value of $0.021 .{ }^{26}$

Dual-Energy X-ray Absorptiometry (DEXA) is used to determine the bone density of lumbar spine, hip, and distal radius. The International Society for Clinical Densitometry recommends bone density testing for all women aged 65 and over, all men aged 70 and older, anyone with a fragility fracture, anyone with a disease, condition or medication associated with osteoporosis, anyone considering therapy for osteoporosis, women who have been on hormone replacement therapy for prolonged periods anyone being treated for osteoporosis and to monitor effects of therapy. DEXA screening should also be considered for younger postmenopausal women with elevated risk, especially women with early menopause and those with a family history of osteoporosis. ${ }^{27}$

BMD is typically expressed in $\mathrm{g} / \mathrm{cm}^{2}$ for which there are different normal ranges for each bone and each type of DXA-measuring machine. The "T score" is a simplified way of reporting bone density in which the patient's bone mineral density is compared to the young normal mean and expressed as a standard deviation score. WHO has established criteria for defining osteoporosis based upon the T score:

- T score $\geq-1.0$ : Normal.

- Tscore-1.0 to-2.5: Osteopenia ("Iow bone density").

- T score <-2.5: Osteoporosis.

- T score $<-2.5$ with a fracture: Severe osteoporosis.

Surveillance DXA bone densitometry is recommended for 
postmenopausal women and elderly men with a frequency according to their T scores: obtain DXA every $10-15$ years for $T$ scores -1.0 to -1.5 , every $3-5$ years for scores -1.5 to -2.0 , and every 2 years for scores under -2.0 or with risk factors that may cause ongoing bone loss. ${ }^{28}$

Several clinical factors are associated with increased fracture risk that cannot be accounted for by BMD alone. ${ }^{29}$ Fracture risk assessment, therefore, should include specific risk factors in addition to BMD.

Fracture Risk Assessment Tool (FRAX) was developed in 2008 by the WHO. FRAX aims to provide an assessment tool for the prediction of fractures in men and women with the use of clinical risk factors with or without femoral neck bone mineral density. These clinical risk factors include age, sex, race, height, weight, body mass index, a history of fragility fracture, parental history of hip fracture, use of oral glucocorticoids, rheumatoid arthritis, and other secondary causes of osteoporosis, current smoking, and alcohol intake of three or more units daily. It is particularly useful for treatment decisions in patients with osteopenia and takes into consideration age, sex, ethnicity, bone mineral density, and other risk factors. Treatment is recommended for individuals with osteopenia ( $T$ score between -1.0 and -2.5) with a computed 10-year hip fracture risk of at least $3 \%$ or a 10 -year risk of any major fracture of at least $20 \% .{ }^{30,31}$ However, the FRAX model has limitations in predicting fracture risk in COPD as it considers only femoral neck bone mineral density and not vertebral bone mineral density which is very common in COPD patients. Assessing the rate of clinical vertebral fracture is also challenging since it is difficult to distinguish between patients with a clinical vertebral fracture and patients who have back pain with an incidental vertebral compression fracture. Therefore, the rates of major osteoporotic fractures reported for sites other than the hip may not be accurate. ${ }^{32}$ Kanis et al. studied the use of clinical risk factors to predict osteoporotic fractures based on baseline and follow-up data from nine population-based cohorts. ${ }^{33}$ They found that models predicted hip fractures substantially better than osteoporotic fractures at other sites, regardless of whether the models used BMD and clinical risk factors alone or a combination of both.

\section{Treatment and Prevention}

The treatment and prevention of osteoporosis in COPD patients require non-pharmacological measures besides pharmacological measures. Respiratory rehabilitation is the chief measure besides smoking cessation which is crucial. Programs like exercise retraining programs improve BMD, muscle strength and balance, and decrease the risk of falling. ${ }^{6}$

Calcium and vitamin D supplementation have proven to be effective in reducing risk of fracture in a dose-dependent manner for vitamin $D$ and only when combined with calcium. ${ }^{34}$ Several recent studies on vitamin D have found that it improves muscle function and postural stability and also have immunomodulatory effects..$^{35,36}$ Daily dose of 800 IU of vitamin D combined with supplemental calcium is recommended for patients with a T-score of,-1 and three minor risk factors (BMI $\leq 21 \mathrm{~kg} / \mathrm{m}^{2}$, chronic alcoholic abuse, active smoking, age $>65$ years, rib fracture, hip fracture, sedentary lifestyle, menopause and $\mathrm{FEV}_{1}<50 \%$ predicted) or one major risk factor (systemic corticosteroid therapy for $>3$ months per year, previous vertebral compression fracture) ${ }^{6}$

\section{Pharmacological Therapy}

Pharmacological treatment is recommended for patients with a spontaneous hip or vertebral fracture or a T score in any location less than or equal to -2.5 . Bisphosphonate therapy has not been specifically evaluated for osteoporosis in COPD patients, although research by SMITH et al. ${ }^{37}$ suggests that it has a beneficial effect on the T-scores of patients with airways disease. The usefulness of new treatments such as teriparatide in COPD remains to be evaluated, whereas its role in the treatment of glucocorticoid-induced osteoporosis now is well established. ${ }^{38}$ Teriparatide has little evidence for hip fracture prevention but seems to be more efficacious on vertebral fractures when compared to oral bisphosphonates, particularly in men. ${ }^{39}$ Selective Estrogen Receptor Modulators (SERMs) such as raloxifene and bazedoxifene may also be used in postmenopausal women who do not have a high risk of hip fractures, since SERMs are effective in the prevention of vertebral fracture but not hip fracture.

Calcitonin: Studies evaluating the effectiveness of calcitonin on glucocorticoid-induced bone loss have been mixed. Luengo et al looked at the effect of nasal calcitonin on bone loss in asthmatic patients who were treated with long-term oral corticosteroid therapy (ie, $10 \mathrm{mg} / \mathrm{d}$ prednisone)..$^{40}$ Those patients receiving calcitonin showed an increase in lumbar spine BMD of $2.8 \%$ at 2 years. Those patients receiving calcium alone showed a loss of BMD of $7.8 \%$ after 2 years. The difference between the groups was statistically significant.

\section{Conclusion}

Pevalence of osteoporosis in COPD ranges from 9-69\% in individuals with COPD. Various risk factors of osteoporosis in COPD patients are older age, emaciation, physical inactivity, vitamin D deficiency, smoking, and systemic inflammation. Osteoporosis-associated fractures, particularly vertebral fracture may further deteriorate pulmonary function and impair daily activities of COPD patients. FRAX and DEXA are non-invasive methods to identify the BMD and to assess the fracture risk in such patients. Therefore, early prevention and treatment of osteoporosis in COPD are 
essential. Effective strategies to prevent bone loss and/ or to treat osteoporosis include calcium and vitamin D, hormone replacement when indicated, calcitonin, and bisphosphonate administration.

\section{Conflict of Interest: None \\ References}

1. Gan WQ, Man SFP, Senthilselvan A et al. Association between chronic obstructive pulmonary disease and systemic inflammation: a systematic review and a meta analysis. Thorax 2004; 59: 574e80.

2. Hansell AL, Walk JA, Soriano JB. What do chronic obstructive pulmonary disease patients die from? A multiple cause coding analysis. Eur Respir J 2003; 22: 809-814.

3. Mannino DM, Watt G, Hole D et al. The natural history of chronic obstructive pulmonary disease. Eur Respir J 2006; 27: 627-643.

4. Mannino DM, Doherty DE, Sonia Buist A. Global Initiative on Obstructive Lung Disease (GOLD) classification of lung disease and mortality: findings from the Atherosclerosis Risk in Communities (ARIC) study. Respir Med 2006; 100: 115-122.

5. Graat-Verboom L, Wouters EFM, Smeenk FW, et al. Current status of research on osteoporosis COPD: a systematic review. Eur Respir J 2009; 34: 209-218.

6. Cavailles A, Brinchault-Rabin G, Dixmier A, Goupil F, Gut-Gobert C, Marchand-Adam S et al. Comorbidities of COPD. Eur Respir Rev 2013; 22(130): 454-475.

7. Scanlon PD, Connett JE, Wise RA et al. Loss of bone density with inhaled triamcinolone in Lung Health Study II. Am J Respir Crit Care Med 2004; 170: 1302-1309.

8. Ferguson GT, Calverley PMA, Anderson JA et al. Prevalence and progression of osteoporosis in patients with COPD. Results from the towards a revolution in COPD Health Study. Chest 2009; 136: 1456-1465.

9. Mineo TC, Ambrogi V, Mineo D et al. Bone mineral density improvement after lung volume reduction surgery for severe emphysema. Chest 2005; 127: 19601966.

10. Maggi S, Siviero P, Gonnelli S et al. EOLO Study Group. Osteoporosis risk in patients with chronic obstructive pulmonary disease: the EOLO study. J Clin Densitom 2009; 12: 345-352.

11. Barnes PJ. Chronic obstructive pulmonary disease: effects beyond the lungs. PLoS Med 2010; 7: e1000220.

12. Janssens $\mathbf{W}$, Bouillon $R$, Claes $B$ et al. Vitamin D deficiency is highly prevalent in COPD and correlates with variants in the vitamin D-binding gene. Thorax 2010; 65: 215-220.

13. Balasubramanian, Vijaya; Naing, Soeb Hypogonadism in chronic obstructive pulmonary disease: incidence and effects, Current Opinion in Pulmonary Medicine:
March 2012 - Volume 18 - Issue 2 - p 112-117 doi: 10.1097/MCP.0b013e32834feb37.

14. Leech JA, Dulberg C, Kellie S et al. Relationship of lung function to severity of osteoporosis in women. Am Rev Respir Dis 1990; 141: 68-71.

15. Lisboa C, Moreno R, Fava M et al. Inspiratory muscle function in patients with severe kyphoscoliosis. Am Rev Respir Dis 1985; 132: 48-52.

16. Myers $A H$, Robinson EG, Van Natta $M L$ et al. Hip fractures among the elderly: factors associated with in-hospital mortality. Am J Epidemiol 1991; 134: 11281137.

17. Shane E, Silverberg SJ, Donovan D et al. Osteoporosis in lung transplantation candidates with end-stage pulmonary disease. Am J Med 1996; 101: 262-269.

18. McEvoy C, Ensrud K, Bender E et al. Association between corticosteroid use and vertebral fractures in older men with chronic obstructive pulmonary disease. Am J Respir Crit Care Med 1998; 157: 704-709.

19. Lyles KW, Gold DT, Shipp KM et al. Association of osteoporotic vertebral compression fractures with impaired functional status. Am J Med 1993; 94: 595601.

20. Nevitt MC, Ettinger B, Black DM et al. The association of radiographically detected vertebral fractures with back pain and function: a prospective study. Ann Intern Med 1998; 128: 793-800.

21. Culham EG, Jimenez HA, King CE. Thoracic kyphosis, rib mobility, and lung volumes in normal women and women with osteoporosis. Spine 1994; 19: 1250-1255.

22. Mussolino ME, Looker AC, Madans JH et al. Risk factors for hip fracture in white men: the NHANES I epidemiologic follow-up study. J Bone Miner Res 1998; 13: 918-924.

23. Van Staa TP, Leufkens HGM, Abenhaim L et al. Use of oral corticosteroids and risk of fractures. J Bone Miner Res 2000; 15: 993-1000.

24. Cooper C, Melton LJ. Magnitude and impact of osteoporosis and fractures. In: Marcus R, Feldman D, Kelsey J, eds. Osteoporosis. New York, NY: Academic Press, 1995; 419-434.

25. NIH Consensus Development Panel on Osteoporosis Prevention D, and Therapy. Osteoporosis prevention, diagnosis, and therapy. JAMA 2001; 285(6): 785-795.

26. Mk D, Bk G, G M, Hs H, T S, D B. Assessment of Osteoporosis and Osteopenia and its Co-Relation with Disease Severity in Patients of Chronic Obstructive Pulmonary Disease with Respect to Associated Risk Factors: A Case Control Study in India. J Pulm Respir Med [Internet] 2018; [cited 2020 Sep 13]; 08(02).

27. 2019 International Society of Clinical Densitometry Official Positions - Adult. Available from: https://www. iscd.org/official-positions/2019-iscd-official-positions- 
adult/. Accessed on $11^{\text {th }}$ October, 2020.

28. Elaine $W$ Yu.Screening for osteoporosis. Available from https://www.uptodate.com/contents/screening-forosteoporosis\#H14. Accessed on 11th October, 2020.

29. Kanis JA. Diagnosis of osteoporosis and assessment of fracture risk. Lancet 2002; 359: 1929-36.

30. National Osteoporosis Foundation. Clinician's guide to prevention and treatment of osteoporosis. 2008. http://www.nof.org/professionals/cliniciansguide_ form.asp. Accessed 2009 Oct 22.

31. Dawson-Hughes B, Tosteson AN, Melton $\sqcup$ 3rd, Baim S, Favus MJ, Khosla S, Lindsay RL; National Osteoporosis Foundation Guide Committee. Implications of absolute fracture risk assessment for osteoporosis practice guidelines in the USA. Osteoporos Int 2008; 19: 449-58.

32. Unnanuntana A, Gladnick BP, Donnelly E, Lane JM. The Assessment of Fracture Risk. J Bone Jt Surg-Am 2010; 92(3): 743-53.

33. Kanis JA, Oden A, Johnell $O$ et al. The use of clinical risk factors enhances the performance of BMD in the prediction of hip and osteoporotic fractures in men and women. Osteoporos Int 2007; 18: 1033-46.

34. Avenell A, Gillespie WJ, Gillespie LD et al. Vitamin D and vitamin $D$ analogs for preventing fractures associated with involutional and post-menopausal osteoporosis. Cochrane Database Syst Rev 2009; 2: CD000227.

35. Buckley LM, Leib ES, Cartularo KS et al. Calcium and vitamin D3 supplementation prevents bone loss in the spine secondary to low-dose corticosteroids in patients with rheumatoid arthritis: a randomized, double-blind, placebo-controlled trial. Ann Intern Med 1996; 125: 961-968.

36. Garcia-Delgado I, Prieto S, Gil-Fraguas L et al. Calcitonin, etidronate, and calcidiol treatment in bone loss after cardiac transplantation. Calcif Tissue Int 1997; 60: 155-159.

37. Smith BJ, Laslett LL, Pile KD et al. Randomized controlled trial of alendronate in airways disease and low bone mineral density. Chron Respir Dis 2004; 1: 131-137.

38. Grossman JM, Gordon R, Ranganath VK et al. American College of Rheumatology 2010 recommendations for the prevention and treatment of glucocorticoid-induced osteoporosis. Arthritis Care Res (Hoboken) 2010; 62: 1515-1526.

39. Chen LX, Zhou ZR, Li YL et al. Comparison of bone mineral density in lumbar spine and fracture rate among eight drugs in treatments of osteoporosis in men: a network meta-analysis. PLoS One 2015; 10(5): e0128032.

40. Luengo $\mathrm{M}$, del Rio L, Pons $\mathrm{F}$ et al. Bone mineral density in asthmatic patients treated with inhaled corticosteroids: a case-control study. Eur Respir J 1997; 10: 2110-2113. 